\title{
Leptospirosis in Bangladesh: An Urgent Need for Coordinated Surveillance System
}

\author{
Shovon Chakma ${ }^{1,2}$ and Sultan Mahmood ${ }^{* 1,2}$ \\ IInstitute of Epidemiology, Disease Control \& Research, Dhaka, Bangladesh; ${ }^{2}$ Massey University, Palmerston North, New Zealand
}

\section{Objective}

To evaluate the necessity of a coordinated leptospira surveillance in Bangladesh through a systematic review.

\section{Introduction}

Fever is a top cause of morbidity in all age groups in Bangladesh and often classified as fever of unknown origin (FUO) in absence of any pathognomonic sign (1). Bangladesh with its long monsoon, frequent flooding, stagnant water, high temperature, humidity and largest density of animal and human population serves as an ideal place for zoonotic transmission of Leptospira (2).

\section{Methods}

We searched three different data bases- PubMed, Web of Science and Google Scholar using the search term Leptospir* OR "Weil's disease" AND Bangladesh which yielded 9, 11 and 2590 articles respectively. We screened the titles first for relevance and later abstracts. Two articles were written in Russian language and the texts were inaccessible. These two articles were not reviewed along with other articles that did not discuss the evidence of leptospira infection in Bangladesh. Five articles met our criteria and were finally chosen for review.

\section{Results}

Leptospirosis was evident in different geographical locations of Bangladesh such as south-eastern, central and north-western part with different landscapes such as highland, plain land and flood prone areas. It was distinctly frequent during bridging period of later winter, start of monsoon, and after monsoon (3-6). Leptospirosis was an eminent cause of fever in urban and rural Bangladesh causing hospitalization $(4,6)$. A study conducted in two hospitals in Dhaka showed $18 \%$ of the dengue-negative patients were positive for leptospirosis (5). Other studies have showed that $2-44 \%$ of febrile outpatients had leptospirosis in Bangladesh $(4,6)$. Furthermore, fever or FUO causes an average of 6.3 work days loss among Bangladeshis (1). Moreover, case fatality rate was higher in leptospirosis $(5 \%)$ than dengue (1.2\%) (5). All these epitomize the necessity of a coordinated leptospirosis surveillance in Bangladesh. A battery of serogroups such as Sarmin, Mini, Australis, Icterhaemorrhagiae, Cynopteri, Autumnalis, Shermani, Javanica, Djasiman, Pyrogenes, Sejroe, Celledoni and Pamana were found in Bangladesh. However, study suggested undifferentiated serovars may be circulating in Bangladesh which resulted in underreporting of Leptospirosis burden (4).

In animal, leptospirosis was common in high yield and pregnant dairy cattle. Farmer's education level, semen source, farm placed at peri-urban area, farm size and employee number were the driving force of leptospirosis at farm level. Serovar Hardjo was found in dairy cattle which confirms the presence of leptospirosis in animal population (7). The allied evidence of leptospirosis in other species as well as risk factors, dynamics of transmission, human animal interfaces, environmental drivers and disease impact can only be generated through a well-established surveillance system. Thus the need of establishment of an immediate surveillance is highlighted to mitigate the risk and disease burden human.

\section{Conclusions}

Our review shows that current study findings do not provide conclusive evidence regarding leptospirosis transmission, circulating serovars and impact in human. Thus a well-coordinated surveillance should be accommodated in the routine surveillance in Bangladesh to alleviate disease risk and morbidity.

\section{Keywords}

Leptospirosis; Surveillance; Bangladesh

\section{References}

1. Statistics BBo. Health and Morbidity Status Survey- 2012. Bangladesh Bureau of Statistics; 2013.

2. Muraduzzaman AKM. Leptospirosis Bangladesh Overview. 4th Global Leptospirosis Action Network Meeting Colombo, Srilanka2014.

3. Morshed M, Konishi H, Terada Y, Arimitsu Y, Nakazawa T. Seroprevalence of leptospirosis in a rural flood prone district of Bangladesh. Epidemiology and infection. 1994;112(03):527-31.

4. Kendall EA, LaRocque RC, Bui DM, Galloway R, Ari MD, Goswami $\mathrm{D}$, et al. Leptospirosis as a cause of fever in urban Bangladesh. The American journal of tropical medicine and hygiene. 2010;82(6):112730.

5. LaRocque RC, Breiman RF, Ari MD, Morey RE, Janan FA, Hayes JM, et al. Leptospirosis during dengue outbreak, Bangladesh. Emerg Infect Dis. 2005;11(5):766-9.

6. Swoboda P, Fuehrer H-P, Ley B, Starzengruber P, Ley-Thriemer K, Jung $\mathrm{M}$, et al. Evidence of a major reservoir of non-malarial febrile diseases in malaria-endemic regions of Bangladesh. The American journal of tropical medicine and hygiene. 2014;90(2):377-82.

7. Parvez M, Prodhan M, Rahman M, Faruque M. Seroprevalence and Associated Risk Factors of Leptospira interrogans Serovar Hardjo in Dairy Cattle of Chittagong, Bangladesh.

\section{*Sultan Mahmood}

E-mail: dr.sultanmahmood@yahoo.com 